\title{
Captación de agua pluvial en un plantel educativo de nivel medio superior del valle de Toluca, México
}

\section{Catchment of pluvial water in a higher middle educational plants in the valley of Toluca, Mexico}

\author{
GÓMEZ-BELTRÁN, Guillermina†* \& PEDROZA-BENÍTEZ, Socorro \\ Universidad Tecnológica del Valle de Toluca, Carrera de Tecnología Ambiental \\ ID 1 er Autor: Guillermina, Gómez-Beltrán / ORC ID: 0000-0002-3621-2262, CVU CONACYT ID: 93419 \\ ID $1^{\text {er }}$ Coautor: Socorro, Pedroza-Benítez / ORC ID: 0000-0002-2745-6994
}

DOI: $10.35429 /$ JCE.2019.7.3.7.11

Recibido 03 de Enero, 2019; Aceptado 30 Marzo, 2019

\begin{abstract}
Resumen
El objetivo del presente proyecto fue diseñar un sistema de captación y tratamiento de aguas pluviales en el Centro de Estudios Tecnológicos Industrial y de Servicio n²3 "Cetis23" como medida correctiva para evitar el encharcamiento de los jardines en temporada de lluvias y aumentar la dotación de agua hacia la población del plantel. El diseño inició a través de un estudio de campo de las superficies de los edificios para determinar cuáles son los que presentan mayor área y mejor superficie, en este caso fueron los edificios con techos a dos aguas. Utilizando el software Sweet Home 3D se simuló el sistema en cada edificio y se realizó un estudio de costos, presupuestando materiales y mano de obra. Se planteó el sistema de tratamiento previo al almacenamiento del agua pluvial que consistió principalmente en un filtro de arena y grava. Finalmente, se diseñó un tanque de almacenamiento de ferrocemento. Este sistema es una opción tecnológica poco compleja, económica y ecológica para dotar de agua, en cantidad y calidad, a la población del CETis 23 (1354 personas con un gasto de $10 \mathrm{~L}$ por persona al día).
\end{abstract}

Captación, Agua Pluvial, Diseño

\begin{abstract}
The objective of this project was to design a rainwater collection and treatment system at the Center for Industrial and Service Technological Studies No. 23 "Cetis23" as a corrective measure to prevent the flooding of gardens in the rainy season and increase the endowment of water to the population of the campus. The design began through a field study of the surfaces of the buildings to determine which are the ones with greater area and better surface, in this case were the buildings with gable roofs. Using the Sweet Home 3D software, the system was simulated in each building and a cost study was carried out, budgeting materials and labor. The pre-storage system for rainwater storage was proposed, consisting mainly of a sand and gravel filter. Finally, a ferrocement storage tank was designed. This system is an uncomplicated, economical and ecological technological option to provide water, in quantity and quality, to the population of CETis 23 (1354 people with an expense of $10 \mathrm{~L}$ per person per day).
\end{abstract}

Catchment, Rainwater, Design

Citación: GÓMEZ-BELTRÁN, Guillermina \& PEDROZA-BENÍTEZ, Socorro. Captación de agua pluvial en un plantel educativo de nivel medio superior del valle de Toluca, México. Revista de Ingeniería Civil. 2019. 3-7: 7-11

\footnotetext{
* Correspondencia del Autor (correo electrónico: guillermina.gomez@utvtol.edu.mx)

$\dagger$ Investigador contribuyendo como primer autor.
} 


\section{Introducción}

El agua es uno de los recursos naturales renovables más importantes para la humanidad y de todos los seres vivos del planeta, su presencia regula el clima y participa de manera importante en la formación de suelos. Hoy en día la sociedad enfrenta graves y complejos problemas relacionados con el agua. La contaminación, deforestación y la sobreexplotación de acuíferos, ha mermado las reservas abastecedoras de muchas ciudades (García, 2012). Los sistemas de captación para uso doméstico y consumo tanto familiar como comunitario son principalmente de dos tipos: los que usan como área de captación los techos y los que usan directamente el suelo.

Los componentes del sistema son básicamente el área de captación, el sistema de conducción, el almacenamiento y el filtro y tratamiento. La cantidad de agua captada está en relación directa con la dimensión del área de captación. Existe un porcentaje de pérdida dependiendo del material con el cual se encuentre elaborada el área de captación y la conducción, se considera un coeficiente de eficiencia entre el $50 \%$ y el $90 \%$ de ahí se puede tomar cualquier valor dependiendo del criterio de quien realice los cálculos. Otro aspecto a tomar en cuenta es si el área de captación posee un ángulo de inclinación el cálculo se debe realizar con las medidas que se obtengan en la proyección vertical de la misma, esto es para efectos de cálculo ya que en caso de que la lluvia llegue con viento puede dar como resultado que caiga con el mismo ángulo de inclinación haciendo efectiva toda el área disponible.

El agua pluvial se recolecta de los techos, suelo, caminos, patios o áreas de captación especialmente preparadas. En el caso de los Techos, los materiales más comunes con los cuales se construyen son: lámina galvanizada, concreto, madera, teja y en algunos casos de láminas de asbesto; En cualquier caso se debe aplicar un tratamiento de impermeabilización, aunque también se puede utilizar todo tipo de superficie impermeable que no desprenda residuos. La condición más importante para las superficies de escurrimiento es que sea impermeable y que permita el escurrimiento, ya que siendo la parte fundamental de este sistema de ella depende la cantidad y calidad del agua captada.
La superficie debe estar limpia, libre de fugas y roturas, preferentemente lisa y uniforme para facilitar el flujo de agua. El presente proyecto tiene como objetivo diseñar un sistema de captación de aguas pluviales en el Centro de Estudios Tecnológicos Industrial y de Servicio No.23 "Cetis23" como medida correctiva para evitar el encharcamiento de los jardines en temporada de lluvias y el aprovechamiento de la misma para uso en servicios sanitarios.

\section{Materiales y métodos}

Se realizó un recorrido de campo por cada uno de los edificios para poder identificar el tipo de losa con el que cuentan e identificar los edificios con mayor área y mejor superficie. Una vez se identificaron los edificios, se desarrolló mediante el software (Sweet Home 3D) cada uno de los diseños de captación. Con este mismo software se diseñaron los cortes y el ensamble de piezas. Posteriormente se eligieron los materiales más adecuados para el sistema de captación y un estudio de costos. Adicionalmente, se planteó un sistema de tratamiento para el tratamiento previo al almacenamiento del agua de lluvia, consistente básicamente en la operación de filtrado y reduciendo los costos al máximo. Finalmente, también se diseñó el tanque de ferocemento mediante el programa de AUTOCAD y a su vez se eligió el edifico más adecuado para colocar un tanque subterráneo.

\section{Resultados y análisis de resultados}

Durante el recorrido de campo se encontraron principalmente dos tipos de edificios (losa plana y a dos aguas). De los edificios que conforman al centro educativo, se eligieron dos edificios, el primero con losa plana y el segundo con losa a dos aguas, debido a que cuentan con mayor área superficial y pendientes que ayudaran a a dirigir el agua captada hacia el punto de recolección. En cuanto al diseño, se elaboró una propuesta de acuerdo al presupuesto del plantel, utilizando materiales de PVC fáciles de conseguir, cuyo costo es menor en comparación de otros materiales, la durabilidad es buena y el mantenimiento es mínimo. Se propuso que las canaletas se construyeran con tubos de PVC cortados longitudinalmente por la mitad, para obtener dos tramos que funcionan como canaletas. 
Para los casos de la edificación con losa plana, el sistema de conducción de las agua hacia un solo punto se dirigirá hacia una bajada exclusiva para aguas pluviales. Partiendo de este punto, las bajadas conducirán las aguas hacia un filtro que permita separar los residuos orgánicos de mayor tamaño para reducir la cantidad de materia orgánica, posteriormente, un sistema de almacenamiento de primeras aguas o filtro y un tanque de almacenamiento final de ferrocemento superficial o enterrado.

Cabe mencionar que el filtro debe ser un elemento indispensable por la cantidad de residuos orgánicos que se llegan a acumular en los techos, puede o no ser un elemento indispensable dependiendo de la cantidad de vegetación que se encuentre en los alrededores de la construcción, si existe algún tipo de vegetación cuyas hojas, frutos o flores se acumulen sobre el techo entonces debe considerarse como indispensable dentro del sistema.

Esta última condición se aplicará en el edificio que se encuentra a las orillas del plantel ya que a los costados de este edificio se encuentra una gran cantidad de árboles, por tal motivo la cantidad de materia orgánica se mayor, y por ende será necesario colocar dicho filtro para así eliminara los componentes orgánicos que pueda acarrear la conducción, estos requisitos son considerados mínimos por la CONAGUA (2016). En el caso de los edificios a dos aguas, el sistema de conducción de agua será a base de canaletas de PVC de $200 \mathrm{~mm}$ de diámetro para evitar una pérdida de agua por desborde, una bajada de aguas pluviales, un sistema de primeras aguas o filtro y serán dirigidas al mismo tanque de almacenamiento propuesto anteriormente.

\section{Diseños de sistemas de captación}

Para el edificio con losa a dos aguas se colocaron las canaletas de PVC en los costados de la losa para poder captar la mayor cantidad de agua y conducirla hacia un solo punto, colocando un recubrimiento para ocultar la tubería (Figura 1).

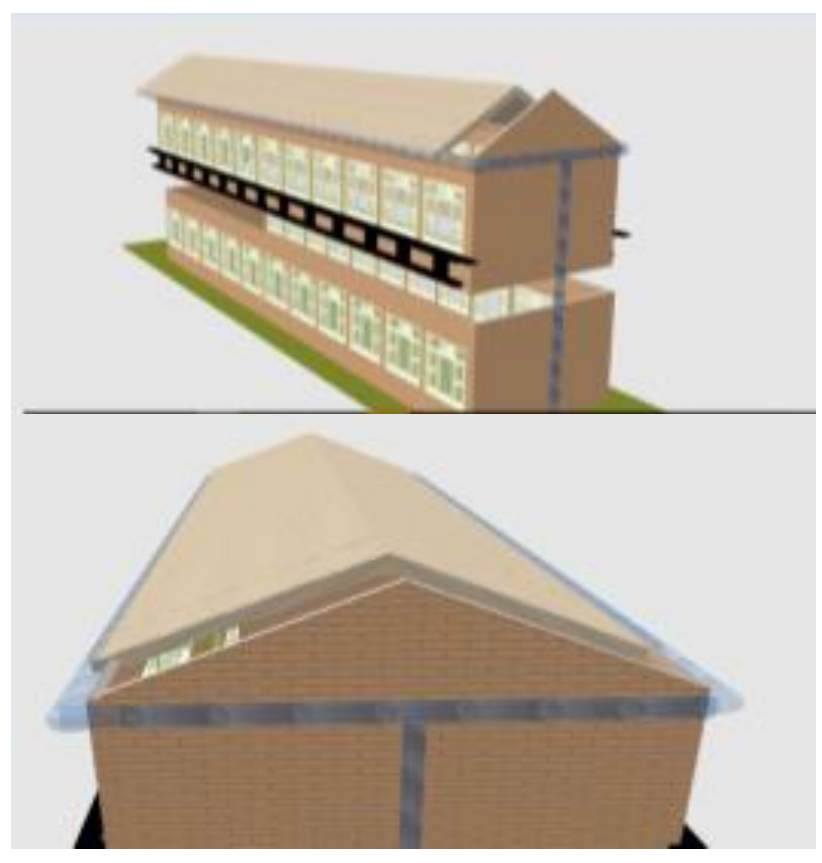

Figura 1 Diseño de captación y condición para edificio de losa a dos aguas

Fuente: Elaboración Propia

De las misma manera, para el edificio con losa plana se hizo el diseño para la captación mediante la tubería de PVC tomando en cuenta un solo lado donde se acumula el agua y posteriormente se conducirá hacia el depósito (Figura 2).

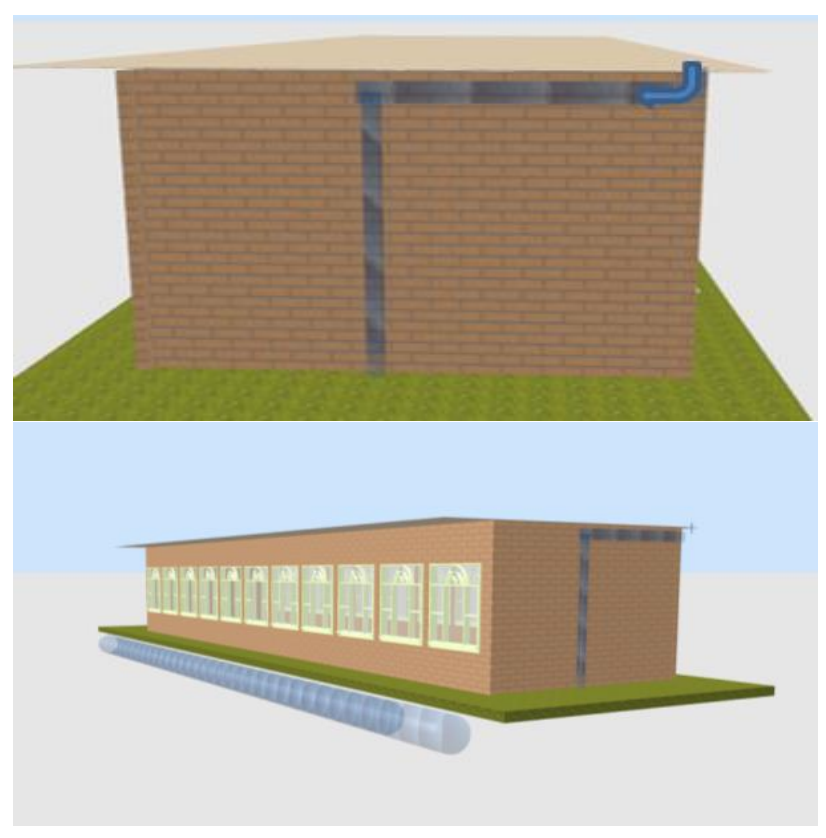

Figura 2 Diseño de captación y condición para edificio de losa plana

Fuente: Elaboración Propia 
En los edificios con techos a dos aguas no es necesario implementar ninguna medida correctiva para hacer eficiente la captación del agua de lluvia, pero con el techo de losa plana, se presenta un acumulamiento de agua que puede llegar a producir filtración al interior del edificio; por ello, se propuso una medida correctiva para conducir el agua hacia un solo punto. La propuesta es colocar una pendiente del $2 \%$ diseñada de tal forma que de los cuatro puntos conduzca el agua hacia la bajada de agua como se muestra en la imagen 2 , el diseño asemeja una hoja de árbol evitando con ello que el agua se escurra por los bordes de la losa (Figura 3).

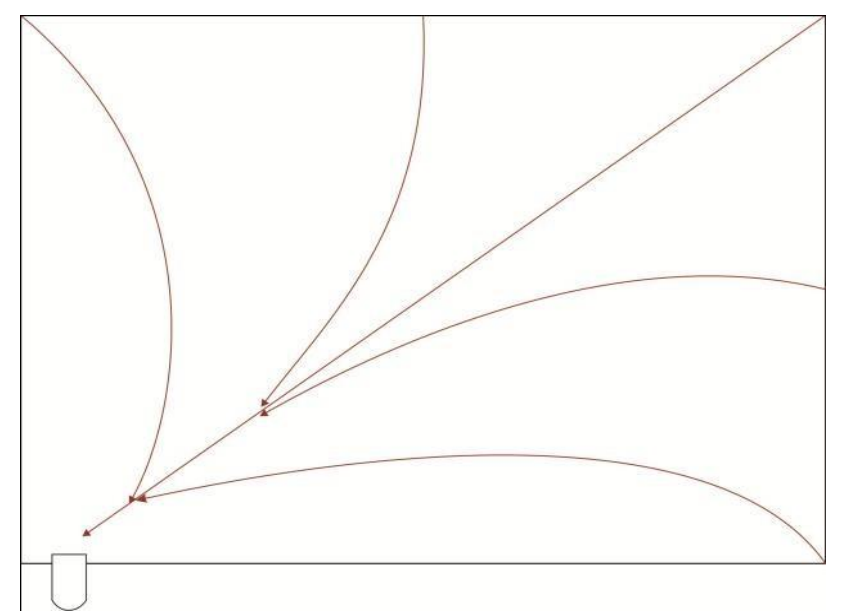

Figura 3 Vista superior de la colocación de la pendiente en losa plan

Fuente: Elaboración Propia

\section{Diseño y colocación del filtro}

El filtro que se propone es con la técnica de ferrocemento, la estructura del filtro lleva $\boldsymbol{e n} \boldsymbol{s u}$ interior una malla cuya función es retener los sólidos asi como controlar la presión con la que llega el agua. La malla es un elemento móvil que puede ser remplazado las veces que sea necesario. Finalmente, el filtro debe rellenarse de abajo hacia arriba con grava, la arena gruesa y por último la arena fina aumentando gradualmente el grosor de las capas como se muestra en la figura 4. Este tipo de filtro se propone para el tanque enterrado ya que funciona por gravedad.

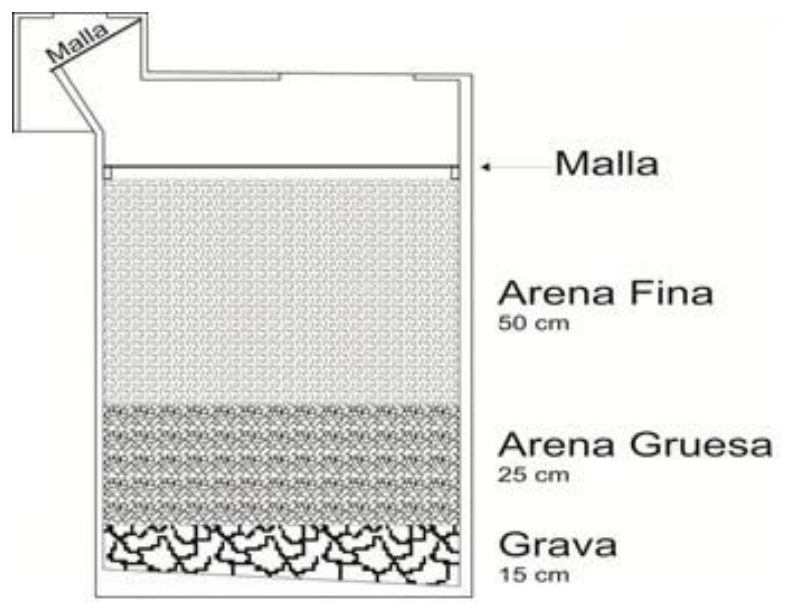

Figura 4 Diseño del filtro para aguas pluviales Fuente: Elaboración Propia

El sistema de primeras aguas se propone elaborar con una serie de tubos como se muestra en la figura 5, la función principal es contener las aguas que tienen la mayor cantidad de contaminantes que el agua atrapa de la atmosfera y del techo que se han acumulado desde la última precipitación, el sistema de funcionamiento es sencillo cuanto más suba el agua, la bola de jebe sube junto con el nivel y al llenarse el recipiente la bola tapa la tubería sellando la entrada y desviándola a la tubería que la lleva al tanque de almacenamiento. El recipiente se propone construido en sitio con ferrocemento, perfil circular con un radio de $35 \mathrm{~cm}$ con una altura de $90 \mathrm{~cm}$ (Figura 5).

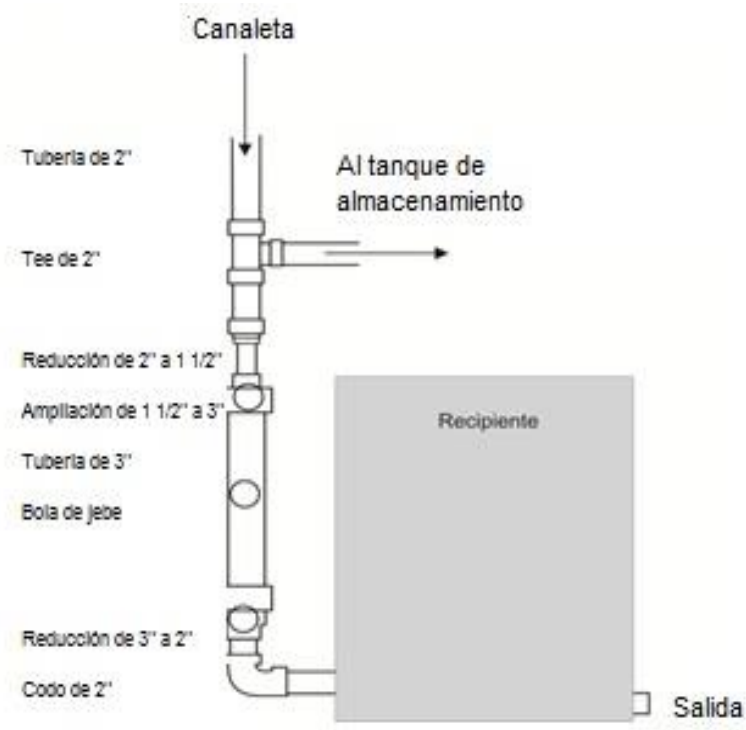

Figura 5 Sistema de primeras aguas Fuente: Elaboración Propia

Para el problema que presenta en el plantel, la meta es recolectar agua para una comunidad de 1,354 personas que se pueda abastecerse durante 3 meses de agua (mayo a julio).

GÓMEZ-BELTRÁN, Guillermina \& PEDROZA-BENÍTEZ, Socorro. Captación de agua pluvial en un plantel educativo de nivel medio superior del valle de Toluca, México. Revista de Ingeniería Civil. 2019 
Por tanto, debe construirse un tanque que contenga 100,000 litros tomando en cuenta un gasto de 10 litros por persona al día, se necesita un volumen de 10 metros cúbicos, si la altura promedio de los tanques de almacenamiento es de 2.5 metros su radio debe ser de $1.2 \mathrm{~m}$.

\section{Diseño de depósito y sistema}

Los materiales propuestos a utilizar para la construcción de depósito de ferrocemento son: cemento, arena con granulometría de 2.15 a 2.75 $\mathrm{mm}$, agua, acero, malla de refuerzo, agregados. El procedimiento de construcción con ferrocemento es sencillo, los pasos principales a seguir son la colocación de la malla de alambre en la posición adecuad, la mezcla de mortero, aplicación del mortero y curado. Cabe mencionar que el tanque de almacenamiento puede manejarse tanto superficial como enterrado y debe tenerse en cuenta que el tanque construido debe contener siempre un mínimo de agua para evitar que se agriete por las altas temperaturas. En el caso del plantel educativo, se optó por un almacenamiento enterrado y resguardado, para evitar accidentes con los alumnos.

\section{Agradecimientos}

Nuestro más sincero agradecimiento al Ing. en Tecnología Ambiental Víctor Hugo Zamora Hidalgo por su valioso trabajo en el desarrollo del presente proyecto.

\section{Conclusiones y recomendaciones}

El sistema de captación de agua de lluvia propuesto presento una opción tecnológica poco compleja, económica y ecológica para abastecer en cantidad y calidad a la población del CETis 23. Considerando que el agua captada será solamente utilizada en el servicio a sanitarios, la propuesta de colocar el filtro es viable ya que con esto se evitara el paso de materia orgánica que pueda tapar, bloquear o dañar el equipo de succión.

En época de lluvias la captación será mayor dado que la precipitación pluvial en valle de Toluca es alta, tomando en cuenta este dato, se espera que en los meses de mayo, junio, y Julio el abastecimiento de agua será suficiente y no se tendrá problemas en cuanto a este recurso para los sanitarios.
El diseño se considera que puede ser implementado a cualquier plantel educativo de la zona de estudio que presente un problema similar, ya que está elaborado con materiales de bajo recurso y se puede conseguir fácilmente.

\section{Referencias}

Comisión Nacional del Agua. (2011). Estadísticas del Agua en México. 15 de junio del 2011. D Recuperado de: http://www.conagua.gob.mx/OCB07/Contenido /Documentos/EstadisticaAguamexico2011.pdf

Comisión Nacional del Agua. (2016). Lineamientos técnicos: sistema de captación de agua de lluvia con fines de abasto de agua potable a nivel vivienda. Recuperado de: https://www.gob.mx/cms/uploads/attachment/fi le/152776/LINEAMIENTOS_CAPTACI_N_P LUVIAL.pdf

García, (2012). Sistema de captación y aprovechamiento pluvial para un ecobarrio de la Cd. de México. (Tesis de Maestría). Recuperado de: http://islaurbana.mx/wpcontent/uploads/2017/06/Captacion-lluviatesisHiram-Garcia.pdf 